
\title{
25 Research Suare \\ Effect of the Amount of Correction on Posterior Tibial Slope and Patellar Height in Open-wedge High Tibial Osteotomy
}

\section{keliang wu}

Guangzhou University of Chinese Medicine

jianchun zeng

Guangzhou University of Traditional Chinese Medicine First Affiliated Hospital

ke jie

Guangzhou University of Chinese Medicine

wenjun feng

Guangzhou University of Traditional Chinese Medicine First Affiliated Hospital

xiaosheng lin

guangzhou medical university

jinlun chen

Guangzhou University of Traditional Chinese Medicine First Affiliated Hospital yirong zeng ( $\boldsymbol{0} 975639330 @ q q . c o m$ )

Guangzhou University of Traditional Chinese Medicine First Affiliated Hospital

\section{Research article}

Keywords: Posterior tibial slope, Patellar height, Open-wedge high tibial osteotomy, Amount of correction, Knee osteoarthritis

Posted Date: April 9th, 2020

DOI: https://doi.org/10.21203/rs.3.rs-19895/v1

License: (c) (1) This work is licensed under a Creative Commons Attribution 4.0 International License. Read Full License

Version of Record: A version of this preprint was published at Journal of Orthopaedic Surgery on September 1st, 2021. See the published version at https://doi.org/10.1177/23094990211049571. 


\section{Abstract}

Objectives The effect of amount of correction on postoperative changes in the posterior tibial slope (PTS), patellar height (PH) and clinical outcomes following biplanar open-wedge high tibial osteotomy (OWHTO) were evaluated.

Method This study included 64 knees (30 left, 34 right) of 64 patients (mean age $48.5 \pm 6.8$ years, 20 males, 44 females) with varus malalignment and symptomatic isolated medial joint osteoarthritis who underwent OWHTO. According to the amount of correction angles, all patients were divided into three groups: large correction angle (LCA) group $\left(>14^{\circ}\right)$, medium correction angle (MCA) group $₫ 10^{\circ} \otimes 14^{\circ}$ \and small correction angle (SCA) group $\left(<10^{\circ}\right)$. All patients were clinically assessed according to the Lysholm score, hospital for special surgery knee score (HSS), and knee society score (KSS) prior to and after surgery. For radiographic analysis, we measured the PTS खPH [Insall-Salvati index (ISI), Blackbume-Peel index (BPI)]. The pre-post difference of PTS, ISI and BPI was calculated by subtracting the post-OWHTO value to the pre-OWHTO value in three groups respectively. The preoperative, postoperative, and difference of PTS, ISI and BPI values were analyzed according to the correction angle. The mean followup period was 27.7 months(SD, 3.9; range 12-49 months).

Results Radiologically, PTS increased and PH decreased after surgery on the whole ( $p$ ه0.05). The relationship between amount of correction and slope increasing is significant ( $p \otimes 0.001)$. Furthermore, the pairwise difference between the LCA group and SCA group, MCA group are significant respectively ( $p$ $\bowtie 0.05)$. In terms of PH, the LCA group yielded ISI and BPI that were significantly different from baseline for SCA group and MCA group. In addition, the pairwise difference between the SCA group and LCA group in ISI and BPI are significant ( $p=0.031)$. Clinically, significant improvements were observed in postoperative clinical scores of the Lysholm score, HSS, and KSS ( $p<0.05)$. Fifty-nine patients $(92.2 \%)$ reported satisfaction with surgery. However, no correlation was found between changes in PTS and PH with postoperative knee score. No severe adverse complications were observed.

Conclusions The amount of correction angle is a significant factor affecting the PTS and PH in OWHTO. With increased correction angle, the likelihood of increasing the PTS and decreasing the PH increases. Special attention should be paid to keep PTS and PH unchanged in cases where large corrections are required. Otherwise, closing wedge osteotomy or other intraoperative effective measures are supposed to be adopted.

\section{Introduction}

Biplanar OWHTO (open-wedge high tibial osteotomy) is one of the most effective surgical procedures in the treatment of isolated medial compartment KOA (knee osteoarthritis) with varus ${ }^{1}$. Many favourable results for OWHTO have recently been reported ${ }^{2-4}$. The main aim of OWHTO is redistribution of load from the medial compartment to the central and lateral areas by correcting the varus deformity of the knee ${ }^{5,6}$. 
Thus, the pressure in medial compartment is decompressed, resulting in pain relieving and delaying of cartilage damage, even partial cartilage regeneration can be observed ${ }^{7,8}$.

Compared with other types of osteotomy surgery, OWHTO has absolute advantages, such as allowing subtle adjustment in the sagittal and coronal planes, avoiding compromising the peroneal nerve $e^{9,10}$. However, some frequently reported problem associated with OWHTO are unintended increasing PTS (posterior tibial slope) and lowering PH (patellar height) ${ }^{11-13}$. Changes of the PH and PTS may result in patellofemoral osteoarthritis and ACL (anterior cruciate ligament) injuries due to increased retropatellar cartilage pressure and higher anteroposterior translation respectively ${ }^{14,15}$.

Factors influencing PTS and PH in OWHTO are thought as follows: the amount of osteotomy opening, degree of knee flexion, ratio between anterior and posterior gap, status of the lateral hinge and the severity of osteoarthritis present in the knee. Despite numerous studies analyse PH and PTS changes after OWHTO $0^{1,2,16-18}$, only a few studies have briefly claimed effect of the amount of osteotomy opening on PTS and PH in OWHTO through cadaveric model ${ }^{19,20}$. Also, data on PTS and PH changes and thus resulted clinical outcomes consequent on various osteotomy opening gap are rare.

To the best of our knowledge, there has been no comparative analysis of PH and PTS changes and knee function between different opening gap after OWHTO in clinic. Therefore, this study is aimed to elucidate the influence of different amount of corrections on the changes of PTS, PH and clinical outcomes in vivo after OWHTO for the first time. It was hypothesized that the amount of correction angle is a significant factor affecting the PTS and PH in OWHTO. With increased correction in the coronal plane, the likelihood of increasing the PTS and decreasing the PH increases.

\section{Materials And Methods}

Between Nov. 2015 to Dec. 2018, all patients undergoing OWHTO were enrolled in this single-center, retrospective study. The study protocol was approved by the ethics committee of hospital institutional review board. The inclusion criteria were as following: (1) patients with symptomatic varus medial joint $\mathrm{OA}$, (2) range of motion $<15^{\circ}$ of flexion contracture, (3) varus aligned knee with $₫ 5^{\circ}$ of mechanical tibiofemoral angle, and (4) medium-to-high level of activity. The Exclusion criteria included (1) lateral and patellofemoral compartments symptoms, (2) previous knee surgery, (3) anterior or posterior cruciate ligament insufficiency with intended slope correction, (4) the presence of rheumatoid arthritis or infectious arthritis, and (5) incomplete or insufficient radiographs.

\section{Surgical procedure}

All osteotomies were performed by one senior orthopedist (Y.R.Z.). Preoperative planning (correction angle and amount of opening gap) was determined as previous described by using the Fujisawa point and Miniaci method ${ }^{21-23}$. 
An oblique skin incision was made from the insertion of the pes anserinus ascending posterocranially aiming at the posteromedial corner of the tibial head. After soft tissue dissection, the superficial portion of the medial collateral ligament was detached distally. A Hohmann retractor was inserted behind the tibial ridge to protect the neurovascular structure. With the leg fully extended, a exact AP view (one third of the fibular head is covered by tibia) of the knee joint was taken under fluoroscopy. Next, the horizontal osteotomy level was defined by two parallel K-wires, which aim towards the proximal tibiofibular joint, approximately $3.5 \mathrm{~cm}-4 \mathrm{~cm}$ below the medial joint line. The intraosseous lengths of two wires were measured, and the depth of the saw cut is $10 \mathrm{~mm}$ less than the value measured against the wires in order to leave a lateral bone hinge. It's important to ensure that there is sufficient space cranial for the locking bolts of the plate fixator. And, special attention should be paid to osteotomize the complete posterior cortex and to release posterior soft tissue in case of hinge fracture.

Afterwards, with knee flexed to $90^{\circ}$, the anterior ascending osteotomy runs at an angle of $110^{\circ}$ to the horizontal saw cut ending behind the patellar tendon insertion. This tuberosity segment should be at least $1.5 \mathrm{~cm} \otimes 2 \mathrm{~cm}$ wide. Using the three-chisel technique, the horizontal osteotomy was gradually opened to desired correction angle ${ }^{24}$.

The medial tibia was fixed with TomoFix ${ }^{\text {TM }}$ Medial High Tibial Plate (Synthes, Solothurn, Switzerland). To minimize tibial slope alteration, the spreader was placed as close to the posterior cortex as possible in addition to the height of the anterior gap of the osteotomy was $60 \%-70 \%{ }^{25,26}$. The appropriate mechnical axis of the lower extremity was checked with a long alignment rod.

\section{Radiographic and knee function assessments}

Preoperative weight-bearing anteroposterior and lateral whole-leg radiographs were obtained to measure PTS, PH and to determine desired correction angle. Also, a skyline view of the knee was taken to evaluate the position changes of patella in coronal plane. The correction angle is defined as angle formed by two lines drawn from the centre of the femoral head and tibiotalar joint to the $62.5 \%$ of the tibial width respectively ${ }^{17}$. According to correction angle, patients were divided into three groups: LCA (large correction angle) group $\left(\triangle 14^{\circ}\right), \mathrm{MCA}$ (medium correction angle) group $\otimes 10^{\circ} \otimes 14^{\circ}$ 囚and SCA (small correction angle) group $\left(\mathbb{}_{10} 0^{\circ}\right.$. PTS was defined as the narrow angle between the proximal tibial anatomic axis and the line tangent to the joint line (Fig.1). The Insall-Salvati index (the ratio between the length of the deep surface of the patellar tendon and the diagonal length of the patella, ISI) and the Blackbume-Peel index (the ratio between perpendicular distance from the inferior tip of patellar articular surface to proximal tibial articular surface and length of the patellar articular surface, BPI) were used to determine the patellar height ${ }^{27,28}$. The pre-post difference of PTS, ISI and BPI was calculated by subtracting the post-OWHTO value to the pre-OWHTO value in three groups respectively. Furthermore, the preoperative, postoperative, and difference of PTS, ISI and BPI values were analyzed according to the correction angle.

All patients' knee condition were clinically evaluated preoperatively and at final follow-up using the Lysholm score, HSS (hospital for special surgery knee score), and KSS (knee society score) through 
clinical follow-up or telephone follow-up. These measurements were performed by a physician independent of the surgical team and blinded to the radiographic findings. The mean follow-up period was 27.7 months(SD, 3.9; range $12 \bigotimes 49$ months).

\section{Statistical analysis}

All radiographic parameters were collected by two experienced orthopaedic surgeons and repeatedly assessed in a blinded fashion 1 months after the first measurement in order to reduce bias. The interclass correlation coefficients for the intra- and inter-observer agreements were calculated. Statistical evaluation was performed using SPSS 22.0 (IBM Corp., Armonk, NY, USA). The continuous data are presented as mean values \pm standard deviation (SD). Student's $t$ test was used to analyze quantitative data, and the chi-squared test was used to analyze qualitative data. The means, SDs, range and differences, comparing the preoperative and postoperative values in the three cohorts, using ANVOA for group comparisons and paired $t$-tests in each group. The $\mathrm{p}$ values of $<0.05$ were considered statistically significant.

\section{Results}

Between Nov. 2015 and Dec. 2018, 64 patients (20 males, 44 females; 64 knees) who underwent OWHTO were included. The mean patient age was 48.5 years (SD, 6.8; range $19 \otimes 77$ years). The mean body mass index was $26.7 \mathrm{~kg} / \mathrm{m}^{2}$ (SD 2.5). Among the 64 knees, 34 (53.1\%) right knees and 30 (46.9\%) left knees were subjected to surgery. To assess the severity of the osteoarthritis in each knee, the K-L (KellgrenLawrence) grade were used. There were 17 knees with grade $\rrbracket, 32$ knees with grade $\otimes$ and 15 knees with grade $\nabla$. The intraobserver reproducibility and interobserver reliability of the $(\mathrm{K}-\mathrm{L})$ grade were 0.903 and 0.879 , respectively. The demographic characteristics of the included population are listed in Table 1 . The interclass correlation coefficients for the intraobserver agreements of the two observer and the interobserver agreement in the values for PTS and PH are showed in Table 2. 
Table 1

Demographics Characteristics

\begin{tabular}{|c|c|c|c|c|c|}
\hline Demographic & $\begin{array}{l}\text { SCA group } \\
\text { ( } n=16 \text { knees) }\end{array}$ & $\begin{array}{l}\text { MCA group } \\
(n=27 \text { knees })\end{array}$ & $\begin{array}{l}\text { LCA group } \\
\text { ( } n=21 \text { knees) }\end{array}$ & $\begin{array}{l}\text { Total } \\
(n=64 \\
\text { knees })\end{array}$ & $\begin{array}{l}\text { P } \\
\text { value }^{\Delta}\end{array}$ \\
\hline $\begin{array}{l}\text { Age } \\
\text { Mean } \pm \text { SD (range) }\end{array}$ & $\begin{array}{l}47.7 \text { years } \\
(7.16) \\
(29 \otimes 55 \text { years })\end{array}$ & $\begin{array}{l}48.9 \text { years } \\
(6.45) \\
(37 \rrbracket 65 \text { years })\end{array}$ & $\begin{array}{l}51.5 \text { years } \\
(8.56) \\
(19 \otimes 77 \text { years })\end{array}$ & $\begin{array}{l}48.5 \text { years } \\
(6.8) \\
(19 \rrbracket \\
77 \text { years) }\end{array}$ & n.s. \\
\hline \multicolumn{6}{|l|}{ Sex } \\
\hline Female & $9(56.3)$ & $16(59.3)$ & $12(57.1)$ & $37(57.8)$ & n.s. \\
\hline Male & $7(43.7)$ & $11(40.7)$ & $9(42.9)$ & $27(42.2)$ & \\
\hline \multicolumn{6}{|l|}{ Side } \\
\hline Right & $9(56.3)$ & $17(63)$ & $13(61.9)$ & $39(60.9)$ & n.s. \\
\hline Left & $7(43.7)$ & $10(37)$ & $8(38.1)$ & $25(39.1)$ & \\
\hline $\mathrm{BMI}(\mathrm{Kg} / \mathrm{m} 2)$ & $25.8 \pm 1.6$ & $26.1 \pm 2.4$ & $26.2 \pm 1.9$ & $26.7 \pm 2.5$ & n.s. \\
\hline Follow-up period, mo & $28.3 \pm 3.1$ & $27.8 \pm 4.2$ & $28.5 \pm 3.8$ & $27.7 \pm 3.9$ & n.s. \\
\hline \multicolumn{6}{|l|}{ Preop K-L grade } \\
\hline ૧ & 5 (31.2) & $7(25.9)$ & $5(23.8)$ & $17(26.6)$ & n.s. \\
\hline प & $7(43.8)$ & $14(51.9)$ & $11(52.4)$ & $32(50)$ & \\
\hline ૧ & $4(25)$ & $6(22.2)$ & $5(28.8)$ & $15(23.4)$ & \\
\hline $\begin{array}{l}\text { Mechanical axis } \\
\text { correction }\end{array}$ & $6.91 \pm 2.31$ & $12.5 \pm 1.31$ & $14.5 \pm 4.5$ & $12.8 \pm 4.9$ & $\nabla 0.001$ \\
\hline \multicolumn{6}{|c|}{$\begin{array}{l}\text { LCA: large correction angle; MCA: medium correction angle; SCA: small correction angle; BMI:Body } \\
\text { mass index; mo: month; K-L grade: the Kellgren and Lawrence grade; } \mathbf{\wedge} \mathrm{P} \text { values are from one-way } \\
\text { ANOVA for continuous variables and from chi-square test for categorical variables. }\end{array}$} \\
\hline
\end{tabular}


Table 2

Intraclass correlation coefficients for intraobserver and interobserver of PTS, ISI and BPI

\begin{tabular}{|c|c|c|c|}
\hline Index & Observer 1 & Observer 2 & Interobserver \\
\hline PTS & $0.853(0.667 \otimes 0.825)$ & $0.894(0.815 \rrbracket 0.932)$ & $0.763(0.577 \rrbracket 0.857)$ \\
\hline \multicolumn{4}{|l|}{$\mathrm{PH}$} \\
\hline Insall-Salvati index & $0.867(0.814 \otimes 0.928)$ & $0.931(0.871 \rrbracket 0.961)$ & $0.838(0.754 \llbracket 0.915)$ \\
\hline Blackbume-Peel index & $0.906(0.923 \otimes 0.968)$ & $0.874(0.847 \rrbracket 0.938)$ & $0.879(0.835 \rrbracket 0.934)$ \\
\hline
\end{tabular}

Mean desired correction angle was $12.87^{\circ}\left(\right.$ range $\left.6^{\circ} \mathrm{Q} 20^{\circ}\right)$; measured postoperative correction angle was $11.92^{\circ}$ (range $5.5^{\circ} 囚 19.7^{\circ}$ ). Radiologically, the effect of correction angle on changes in PTS and PH were showed in Table 3. The LCA group yielded tibial slopes that were significantly different from SCA group and MCA group. The mean difference of PTS for LCA group was $3.07 \pm 2.16$. The difference from baseline PTS is smallest for the SCA group $(0.38 \pm 1.21)$ and greatest for the LCA group. In terms of PH, the LCA group yielded ISI and BPI that were significantly different from baseline for SCA group and MCA group. The mean difference of ISI and BPI for LCA group were $0.17 \pm 0.21$ and $0.17 \pm 0.17$ respectively. The difference from baseline ISI and BPI all are smallest for the SCA group $(0.03 \pm 0.24,0.01 \pm 0.19$, respectively) and greatest for the LCA group. 
Table 3

Preoperative and Postoperative Radiological Evaluations

\begin{tabular}{|c|c|c|c|c|}
\hline Variable & $\begin{array}{l}\text { SCA group } \\
(n=16 \\
\text { knees) }\end{array}$ & $\begin{array}{l}\text { MCA group } \\
(n=27 \\
\text { knees) }\end{array}$ & $\begin{array}{l}\text { LCA group } \\
(n=21 \\
\text { knees })\end{array}$ & $\begin{array}{l}\mathrm{P} \\
\text { value }^{4}\end{array}$ \\
\hline \multicolumn{5}{|l|}{ Tibial slope [Mean \pm SD (range)] } \\
\hline Preoperative & $7.81 \pm 1.52$ & $7.91 \pm 1.72$ & $8.23 \pm 1.63$ & 0.698 \\
\hline Postoperative & $8.18 \pm 2.33$ & $9.09 \pm 2.49$ & $11.3 \pm 2.86$ & 0.001 \\
\hline $\begin{array}{l}\text { Change for postoperative from } \\
\text { preoperative }\end{array}$ & $0.38 \pm 1.21$ & $1.18 \pm 1.6$ & $3.07 \pm 2.16$ & 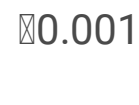 \\
\hline p value $\mathbf{\Lambda \Lambda}^{\Delta}$ & 0.233 & 0.001 & $\varangle 0.001$ & \\
\hline \multicolumn{5}{|c|}{ Insall-Salvati index [Mean \pm SD (range)] } \\
\hline Preoperative & $0.96 \pm 0.15$ & $0.96 \pm 0.1$ & $0.97 \pm 0.17$ & 0.965 \\
\hline Postoperative & $0.93 \pm 0.19$ & $0.83 \pm 0.14$ & $0.76 \pm 0.11$ & 0.004 \\
\hline $\begin{array}{l}\text { Change for postoperative from } \\
\text { preoperative }\end{array}$ & $-0.03 \pm 0.24$ & $-0.12 \pm 0.17$ & $-0.21 \pm 0.2$ & 0.03 \\
\hline p value $\mathbf{\Lambda \Lambda}$ & 0.398 & $\varangle 0.001$ & $\otimes 0.001$ & \\
\hline \multicolumn{5}{|l|}{$\begin{array}{l}\text { Blackbume-Peel index [Mean } \pm \text { SD } \\
\text { (range)] }\end{array}$} \\
\hline Preoperative & $0.76 \pm 0.14$ & $0.77 \pm 0.13$ & $0.77 \pm 0.14$ & 0.935 \\
\hline Postoperative & $0.75 \pm 0.21$ & $0.64 \pm 0.15$ & $0.6 \pm 0.12$ & 0.025 \\
\hline $\begin{array}{l}\text { Change for postoperative from } \\
\text { preoperative }\end{array}$ & $-0.01 \pm 0.19$ & $-0.13 \pm 0.18$ & $-0.17 \pm 0.17$ & 0.031 \\
\hline p value $\mathrm{A}^{\mathbf{\Delta}}$ & 0.837 & 0.001 & $\varangle 0.001$ & \\
\hline
\end{tabular}

The effect of amount of correction on change in PTS was analyzed. The relationship between amount of correction and slope is significant ( $p \bigotimes 0.001)$. The pairwise difference between the LCA group and the SCA group, MCA group were significant ( $p \bigotimes 0.05)$. However, the difference in SCA group and MCA group had no significance ( $p=0.435)$ (Fig. 2).

The effect of amount of correction on change in $\mathrm{PH}$ was also analyzed. The relationship between amount of correction and ISI, BPI were significant respectively $(p=0.03, p=0.031)$. The pairwise difference between the SCA group and LCA group in ISI and BPI are significant $(p=0.031)$; however, the pairwise 
difference between the SCA group and MCA group and between MCA group and LCA group had no significance in ISI and BPI respectively ( $p \rrbracket)$ (Fig. 3) (Fig. 4).

Clinically, the preoperative mean Lysholm score, HSS score and KSS score of the knee all had improved substantially after surgery (Table 4). Furthermore, there were significant improvements of knee scores in each three groups respectively, which corresponded to functional results that were excellent in 33 knees, good in 15 knees, fair in 13 knees, and poor in three knees. No correalation was found between changes in PTS and PH with postoperative Lysholm score, HSS score and KSS score. Fifty-nine patients (92.2\%) reported satisfaction with surgery.

Table 4

Preoperative and Postoperative Clinical Evaluations

\begin{tabular}{|c|c|c|c|c|c|}
\hline Variable & $\begin{array}{l}\text { SCA group } \\
(n=16 \\
\text { knees) }\end{array}$ & $\begin{array}{l}\text { MCA group } \\
(n=27 \\
\text { knees) }\end{array}$ & $\begin{array}{l}\text { LCA group } \\
(n=21 \\
\text { knees) }\end{array}$ & $\begin{array}{l}\text { Total } \\
(n=64 \\
\text { knees })\end{array}$ & $\begin{array}{l}\mathrm{P} \\
\text { value }^{\Delta}\end{array}$ \\
\hline \multicolumn{6}{|c|}{$\begin{array}{l}\text { Lysholm score (Mean } \pm \\
\text { SD) }\end{array}$} \\
\hline Preoperative & $57.6 \pm 4.9$ & $56.5 \pm 5.3$ & $56.1 \pm 5.5$ & $56.7 \pm 5.24$ & 0.675 \\
\hline Last follow up & $88.1 \pm 3.7$ & $87.1 \pm 5.5$ & $86.6 \pm 4.9$ & $87.17 \pm 4.88$ & 0.582 \\
\hline $\mathrm{p}$ value $\mathbf{\Lambda} \boldsymbol{\Lambda}$ & $\varangle 0.001$ & $\varangle 0.001$ & $\varangle 0.001$ & $\otimes 0.001$ & \\
\hline \multicolumn{6}{|c|}{ HSS score (Mean \pm SD) } \\
\hline Preoperative & $61.1 \pm 4.7$ & $60.2 \pm 7$ & $59.9 \pm 5.6$ & $60.33 \pm 5.95$ & 0.84 \\
\hline Last follow up & $88.9 \pm 5.5$ & $88.3 \pm 6.2$ & $87.7 \pm 5.4$ & $88.28 \pm 5.71$ & 0.815 \\
\hline p value $\mathrm{A}^{\mathbf{\Lambda}}$ & $\varangle 0.001$ & $凶 0.001$ & $\bowtie 0.001$ & $\llbracket 0.001$ & \\
\hline \multicolumn{6}{|c|}{ KSS score (Mean \pm SD) } \\
\hline Preoperative & $58.9 \pm 6.5$ & $57.8 \pm 6.6$ & $56.9 \pm 5.1$ & $57.77 \pm 6.1$ & 0.614 \\
\hline Last follow up & $83.5 \pm 7.8$ & $81.2 \pm 7.7$ & $81.6 \pm 6.7$ & $81.92 \pm 7.36$ & 0.609 \\
\hline p value $\mathbf{\Lambda \Delta}$ & $\varangle 0.001$ & $\varangle 0.001$ & $\varangle 0.001$ & $\varangle 0.001$ & \\
\hline
\end{tabular}

With regard to complications, four patients developed non-displaced fractures within the tibiofibular joint

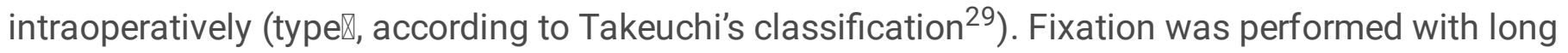
metaphyseal cancellous bone screws followed by 4 weeks of delayed postoperative mobilization 
exercises. Finally, these patients were observed without obvious functional deficit. Two other patients developed superficial infections, which resolved completely with intravenous antibiotics therapy and wound dressing change.

\section{Discussion}

The most important finding of the present study is that correction angle was a significant factor affecting the PTS and PH in patients who underwent OWHTO. The changes are highly variable and often the amount of change is too small to be clinically significant; however, the maximum assessed changes during this study (maximal PTS change $12^{\circ}$, maximal ISI change 0.9 and maximal BPI change 0.75 ) highlight the need to be vigilant for these problems.

To our knowledge, this is the first clinical study evaluating the effect of different amount of correction on PTS and PH in OWHTO to date. PTS is important for knee extension and flexion, for the correct function of the cruciate ligaments and normal knee kinematics ${ }^{30}$. In OWHTO,several factors could influence PTS, including the amount of correction ${ }^{20}$, optimal gap ratio in the sagittal plane ${ }^{31-33}$, hinge position ${ }^{31,32}$, hinge fracture, insufficient posterior osteotomy and release of soft tissue ${ }^{31,34}$. Many studies have demonstrated an increased PTS after OWHTO, 17,20,35-37.

In our study, we observed that the mean postoperative PTS increased. The change of PTS in MCA group and LCA group showed a significant difference compared with preoperative PTS. And, LCA group yielded tibial slopes that were significantly different from SCA group and MCA group, which indicates that the amount of correction performed is one of the key factors affecting the PTS after OWHTO. It is clear that the larger the correction, the more freedom is present for PTS or PH. Matthias et al. ${ }^{20}$ evaluated the influence of certain intraoperative factors of the degree of PTS change observed during OWHTO through a cadaveric model. They found that larger corrections yielded not only increased mean values for PTS, but also increased variability in the observed values. The changes noted in small corrections were generally below clinical significance; however, in cases with concomitant ACL deficiency, even small changes may be important. This result is consistent with another cadaveric study conducted by Rubino et al. ${ }^{19}$. El-Azab et al. ${ }^{38}$ found that PTS increased after OWHTO because of the geometry of the proximal tibial, which dictates corresponding sagittal plane changes in coronal plane ostetomies. In a recent metaanalysis, an unintentional mean postoperative slope increase of $2.02^{\circ}$ has been detected in $\mathrm{OWHTO}^{39}$.

It has been suggested that increased PTS may result in knee instability and tibial translation including increased ACL strain, posteriorly shifted intra-articular cartilage peak pressure. In addition, those patients with increased PTS often underwent more bone loss of the tibial plateau posteriorly, which could make it more difficult to possible future total knee arthroplasty $(T K A)^{40}$. However, the incorporation of specific surgical techniques such as controlling anterior/posterior osteotomy opening gap ratio between $2: 3^{41}$; a complete osteotomy of the posterior corte ${ }^{42}$, putting the plate as far posteriorly as possible ${ }^{43}$, using reference K-wires for intra-operative PTS orientation ${ }^{44}$, applying a bicortical screw to fix the distal 
tuberosity to the tibia ${ }^{45}$ and avoiding larger corrections ${ }^{43}$ may helpful in minimizing PTS change. Nevertheless, of course,such an increased PTS can be beneficial in cases of posterior cruciate ligament deficiency, as it can lead to anterior translation of the tibial relative to the femur, especially if ACL deficiency is present ${ }^{46}$.

Ideally, any surgery on the knee should not significantly worsen the position of the patella, making more attention should be paid to avoid patellofemoral issues. A normal PH is an important determinant for knee function, otherwise, may result in anterior knee pain, decreased range of motion, patellofemoral arthrosis, and potentially complicated future TKA ${ }^{9,35}$. However, we observed that the mean postoperative patellar height decreased after OWHTO in our study. The mean change in ISI and BPI between the SCA group and LCA group are significant ( $p \varangle 0.05)$, thus we speculated that larger correction angle seems to decrease the patellar position and may have a negative impact on patellar height. In OWHTO, due to the attachment of the tuberosity to the distal fragment, the tibial insertion of the patellar is distalized. Also, a potential post-operative tendon shortening caused by scarring may occur.

Hence, in order to avoid a PH decrease, Gaasbeek et al. ${ }^{47}$ introduced a biplanar descending OWHTO, of which sagittal osteotomy line was below the tibial tuberosity. Krause et al. ${ }^{2}$ conducted a study to gain insight into geometric changes of the PH and PTS after biplanar ascending OWHTO compared to descending OWHTO in patients with KOA. They found that descending OWHTO has proven useful to control PH and PTS, which is in line with other previous results ${ }^{44,48-50}$. However, this techniques are technically demanding and could lead to the potential for fracture of tibial tubercle fragment. Park et al. ${ }^{51}$ found two tibial tuberosity fractures with descending OWHTO in 33 patients, but they noted that this complication is preventable by leaving more than $1 \mathrm{~cm}$ thickness on the proximal tuberosity fragment.

There was a significant increase in the clinical score after OWHTO. Overall, OWHTO is an effective treatment for isolated medial compartment KOA in varus knees. Patients reported pain relief, improvement in whole-knee function and satisfaction with the clinical outcome. NO patellofemoral joint symptoms or other uncomfortable symptoms were observed. Although increased PTS and descent of the patella were observed, postoperative knee scores showed improvement compared with preoperative values, indicating changes of PTS and PH had no meaningful effect on clinical result in short term. In addition, there were no significant difference in terms of postoperative knee scores among three groups, suggesting the effect of different correction angles on knee function and pain was limited.

This study was not without limitations. First, the sample was relatively small and the follow-up was relatively short. Large sample size research and long-term follow-up were required to further evaluate the effect of correction angles on knee joint. Second, the slope was measured only with plain radiographs, and this may predispose to measurement error. But, two experienced observers evaluated radiographic parameters twice in a blinded fashion, which could possible to minimize potential bias from this limitation. Third, no single method of measuring PH has been accepted as the gold standard so far. Each method has it's own particular limitations, which is probably why numerous previous studies have used two or three methods. 


\section{Conclusion}

OWHTO is an effective procedure to unload the medial compartment of knee joint. The amount of correction angle is a significant factor affecting the PTS and PH in OWHTO clinically, although no association was found between those unintended changes and worsening knee clinical symptoms in short term. With increased correction in the coronal plane, the likelihood of increasing the PTS and decreasing the $\mathrm{PH}$ increases. We conclude that special attention should be paid to keep PTS and PH unchanged in cases where large corrections are required. Otherwise, closing wedge osteotomy or other intraoperative effective measures are supposed to be adopted.

\section{Abbreviations}

OWHTO:open-wedge high tibial osteotomy; KOA:knee osteoarthritis; PTS:posterior tibial slope; PH:patellar height; ACL:anterior cruciate ligament; LCA:large correction angle; MCA:medium correction angle; SCA:small correction angle; HSS:hospital for special surgery knee score; KSS:knee society score; ISI:Insall-Salvati index; BPI:Blackbume-Peel index; K-L:Kellgren-Lawrence

\section{Declarations}

\section{Acknowledgements}

This study was supported by the Third Department of Orthopedics, The First Affiliated Hospital of Guangzhou University of Chinese Medicine. However, the interpretation and conclusions contained in this study are those of the authors alone.

\section{Authors'contributors}

YRZ conceived the study. KLW acquired the data. JCZ, KJ, WJF, and XSL developed the analysis plan. XSL and JLC analyzed the data. JCZ and KLW drafted the manuscript. KLW, JCZ, KJ, XSL, WJF, JLC and YRZ revised the manuscript. All authors contributed intellectually to the interpretation of the data, participated in manuscript development, and approved the final version. YRZ is the guarantor.

\section{Funding}

We received no financial support for the research and/or authorship of this article.

\section{Availability of data and materials}

No additional data are available.

\section{Ethics approval and consent to participate}

Approval was granted by the First Affiliated Hospital of Guangzhou University of Chinese Medicine. 


\section{Consent for publication}

Not applicable.

\section{Competing interests}

The authors declare that they no conflicts of interest.

\section{Author details}

${ }^{1}$ School of Shenzhen Bao'An Shajing People's Hospital, Guangzhou University of Chinese Medicine, 12nd Jichang Road, Baiyun District, Guangzhou, Guangdong Province 510405, China; ${ }^{2}$ The Third Department of Orthopedics, The First Affiliated Hospital of Guangzhou University of Chinese Medicine, 16th Jichang Road, Baiyun District, Guangzhou, Guangdong Province 510405, China; ${ }^{3}$ The First Clinical Medical College, Guangzhou University of Chinese Medicine, 12nd Jichang Road, Baiyun District, Guangzhou, Guangdong Province 510405, China; ${ }^{4}$ ShenZhen Bao'An Shajing People's Hospital, Guangzhou Medical University, 3rd Shajin Road, Bao'an District, Shenzhen, Guangdong Province, 518104, China.

\section{References}

1. Jingbo C, Mingli F, Guanglei C, Zheng L, Shuai A, Jiang H. Erratum to: Patellar Height Is Not Altered When the Knee Axis Correction Is Less than 15 Degrees and Has Good Short-Term Clinical Outcome. J Knee Surg, 2019.

2. Krause M, Drenck TC, Korthaus A, Preiss A, Frosch KH, Akoto R. Patella height is not altered by descending medial open-wedge high tibial osteotomy (HTO) compared to ascending HTO. Knee Surg Sports Traumatol Arthrosc, 2018, 26: 1859-1866.

3. Saito T, Kumagai K, Akamatsu Y, Kobayashi H, Kusayama Y. Five- to ten-year outcome following medial opening-wedge high tibial osteotomy with rigid plate fixation in combination with an artificial bone substitute. Bone Joint J, 2014, 96-B: 339-344.

4. Souza $L$, Rocha V, Ramos M. The role of microfractures with tibial osteotomy in the treatment of knee osteoarthritis with a varus deformity. Rev Bras Ortop, 2018, 53: 754-760.

5. Akizuki S, Shibakawa A, Takizawa T, Yamazaki I, Horiuchi H. The long-term outcome of high tibial osteotomy: a ten- to 20-year follow-up. J Bone Joint Surg Br, 2008, 90: 592-596.

6. Arun GR, Kumaraswamy V, Rajan D, et al. Long-term follow up of single-stage anterior cruciate ligament reconstruction and high tibial osteotomy and its relation with posterior tibial slope. Arch Orthop Trauma Surg, 2016, 136: 505-511.

7. Bito H, Takeuchi R, Kumagai K, et al. Opening wedge high tibial osteotomy affects both the lateral patellar tilt and patellar height. Knee Surg Sports Traumatol Arthrosc, 2010, 18: 955-960.

8. Bode G, von Heyden J, Pestka J, et al. Prospective 5-year survival rate data following open-wedge valgus high tibial osteotomy. Knee Surg Sports Traumatol Arthrosc, 2015, 23: 1949-1955. 
9. Agneskirchner JD, Hurschler C, Stukenborg-Colsman C, Imhoff AB, Lobenhoffer P. Effect of high tibial flexion osteotomy on cartilage pressure and joint kinematics: a biomechanical study in human cadaveric knees. Winner of the AGA-DonJoy Award 2004. Arch Orthop Trauma Surg, 2004, 124: 575584.

10. Brouwer RW, Bierma-Zeinstra SM, van Koeveringe AJ, Verhaar JA. Patellar height and the inclination of the tibial plateau after high tibial osteotomy. The open versus the closed-wedge technique. J Bone Joint Surg Br, 2005, 87: 1227-1232.

11. LaPrade RF, Oro FB, Ziegler CG, Wijdicks CA, Walsh MP. Patellar height and tibial slope after openingwedge proximal tibial osteotomy: a prospective study. Am J Sports Med, 2010, 38: 160-170.

12. Cantin O, Magnussen RA, Corbi F, Servien E, Neyret P, Lustig S. The role of high tibial osteotomy in the treatment of knee laxity: a comprehensive review. Knee Surg Sports Traumatol Arthrosc, 2015, 23 : 3026-3037.

13. Cotic $M$, Vogt $S$, Hinterwimmer $S$, et al. A matched-pair comparison of two different locking plates for valgus-producing medial open-wedge high tibial osteotomy: peek-carbon composite plate versus titanium plate. Knee Surg Sports Traumatol Arthrosc, 2015, 23: 2032-2040.

14. Feucht MJ, Mauro CS, Brucker PU, Imhoff AB, Hinterwimmer S. The role of the tibial slope in sustaining and treating anterior cruciate ligament injuries. Knee Surg Sports Traumatol Arthrosc, 2013, 21: 134-145.

15. McLean SG, Oh YK, Palmer ML, et al. The relationship between anterior tibial acceleration, tibial slope, and ACL strain during a simulated jump landing task. J Bone Joint Surg Am, 2011, 93: 13101317.

16. Portner $\mathrm{O}$. High tibial valgus osteotomy: closing, opening or combined? Patellar height as a determining factor. Clin Orthop Relat Res, 2014, 472: 3432-3440.

17. Ozel O, Yucel B, Mutlu S, Orman O, Mutlu H. Changes in posterior tibial slope angle in patients undergoing open-wedge high tibial osteotomy for varus gonarthrosis. Knee Surg Sports Traumatol Arthrosc, 2017, 25: 314-318.

18. Akamatsu $\mathrm{Y}$, Kumagai $\mathrm{K}$, Kobayashi $\mathrm{H}$, Tsuji M, Saito T. Effect of Increased Coronal Inclination of the Tibial Plateau After Opening-Wedge High Tibial Osteotomy. Arthroscopy, 2018, 34: 2158-2169.e2.

19. Rubino LJ, Schoderbek RJ, Golish SR, Baumfeld J, Miller MD. The effect of plate position and size on tibial slope in high tibial osteotomy: a cadaveric study. J Knee Surg, 2008, 21: 75-79.

20. Jacobi M, Villa V, Reischl N, et al. Factors influencing posterior tibial slope and tibial rotation in opening wedge high tibial osteotomy. Knee Surg Sports Traumatol Arthrosc, 2015, 23: 2762-2768.

21. Fujisawa Y, Masuhara K, Shiomi S. The effect of high tibial osteotomy on osteoarthritis of the knee. An arthroscopic study of 54 knee joints. Orthop Clin North Am, 1979, 10: 585-608.

22. Elson DW, Petheram TG, Dawson MJ. High reliability in digital planning of medial opening wedge high tibial osteotomy, using Miniaci's method. Knee Surg Sports Traumatol Arthrosc, 2015, 23: 2041 2048. 
23. Han SB, Lee DH, Shetty GM, Chae DJ, Song JG, Nha KW. A "safe zone" in medial open-wedge high tibia osteotomy to prevent lateral cortex fracture. Knee Surg Sports Traumatol Arthrosc, 2013, 21: 9095.

24. Staubli AE, De Simoni C, Babst R, Lobenhoffer P. TomoFix: a new LCP-concept for open wedge osteotomy of the medial proximal tibia-early results in 92 cases. Injury, 2003, 34 Suppl 2: B55-62.

25. Chae DJ, Shetty GM, Lee DB, Choi HW, Han SB, Nha KW. Tibial slope and patellar height after opening wedge high tibia osteotomy using autologous tricortical iliac bone graft. Knee, 2008, 15: 128-133.

26. Lee SY, Lim HC, Bae JH, et al. Sagittal osteotomy inclination in medial open-wedge high tibial osteotomy. Knee Surg Sports Traumatol Arthrosc, 2017, 25: 823-831.

27. Caton J, Deschamps G, Chambat P, Lerat JL, Dejour H. [Patella infera. Apropos of 128 cases]. Rev Chir Orthop Reparatrice Appar Mot, 1982, 68: 317-325.

28. Insall J, Salvati E. Patella position in the normal knee joint. Radiology, 1971, 101: 101-104.

29. Takeuchi $R$, Ishikawa $H$, Kumagai $K$, et al. Fractures around the lateral cortical hinge after a medial opening-wedge high tibial osteotomy: a new classification of lateral hinge fracture. Arthroscopy, 2012, 28: 85-94.

30. Amis AA. Biomechanics of high tibial osteotomy. Knee Surg Sports Traumatol Arthrosc, 2013, 21: 197-205.

31. Wang JH, Bae JH, Lim HC, Shon WY, Kim CW, Cho JW. Medial open wedge high tibial osteotomy: the effect of the cortical hinge on posterior tibial slope. Am J Sports Med, 2009, 37: 2411-2418.

32. Moon SW, Park SH, Lee BH, et al. The Effect of Hinge Position on Posterior Tibial Slope in Medial Open-Wedge High Tibial Osteotomy. Arthroscopy, 2015, 31: 1128-1133.

33. Ogawa H, Matsumoto K, Ogawa T, Takeuchi K, Akiyama H. Effect of Wedge Insertion Angle on Posterior Tibial Slope in Medial Opening Wedge High Tibial Osteotomy. Orthop J Sports Med, 2016, 4: 2325967116630748.

34. Marti CB, Gautier E, Wachtl SW, Jakob RP. Accuracy of frontal and sagittal plane correction in openwedge high tibial osteotomy. Arthroscopy, 2004, 20: 366-372.

35. El-Azab H, Glabgly P, Paul J, Imhoff AB, Hinterwimmer S. Patellar height and posterior tibial slope after open- and closed-wedge high tibial osteotomy: a radiological study on 100 patients. Am J Sports Med, 2010, 38: 323-329.

36. Ducat A, Sariali E, Lebel B, et al. Posterior tibial slope changes after opening- and closing-wedge high tibial osteotomy: a comparative prospective multicenter study. Orthop Traumatol Surg Res, 2012, 98 : 68-74.

37. Bae DK, Ko YW, Kim SJ, Baek JH, Song SJ. Computer-assisted navigation decreases the change in the tibial posterior slope angle after closed-wedge high tibial osteotomy. Knee Surg Sports Traumatol Arthrosc, 2016, 24: 3433-3440. 
38. El-Azab H, Halawa A, Anetzberger H, Imhoff AB, Hinterwimmer S. The effect of closed- and openwedge high tibial osteotomy on tibial slope: a retrospective radiological review of 120 cases. J Bone Joint Surg Br, 2008, 90: 1193-1197.

39. Nha KW, Kim HJ, Ahn HS, Lee DH. Change in Posterior Tibial Slope After Open-Wedge and ClosedWedge High Tibial Osteotomy: A Meta-analysis. Am J Sports Med, 2016, 44: 3006-3013.

40. Hernigou P, Medevielle D, Debeyre J, Goutallier D. Proximal tibial osteotomy for osteoarthritis with varus deformity. A ten to thirteen-year follow-up study. J Bone Joint Surg Am, 1987, 69: 332-354.

41. Song EK, Seon JK, Park SJ. How to avoid unintended increase of posterior slope in navigationassisted open-wedge high tibial osteotomy. Orthopedics, 2007, 30: S127-131.

42. Lobenhoffer $P$, Agneskirchner JD. Improvements in surgical technique of valgus high tibial osteotomy. Knee Surg Sports Traumatol Arthrosc, 2003, 11: 132-138.

43. Jung KA, Kim SJ, Lee SC, Song MB, Yoon KH. 'Fine-tuned' correction of tibial slope with a temporary external fixator in opening wedge high-tibial osteotomy. Knee Surg Sports Traumatol Arthrosc, 2008, 16: 305-310.

44. Hinterwimmer S, Beitzel K, Paul J, et al. Control of posterior tibial slope and patellar height in openwedge valgus high tibial osteotomy. Am J Sports Med, 2011, 39: 851-856.

45. Faul F, Erdfelder E, Lang AG, Buchner A. G*Power 3: a flexible statistical power analysis program for the social, behavioral, and biomedical sciences. Behav Res Methods, 2007, 39: 175-191.

46. Giffin JR, Vogrin TM, Zantop T, Woo SL, Harner CD. Effects of increasing tibial slope on the biomechanics of the knee. Am J Sports Med, 2004, 32: 376-382.

47. Gaasbeek RD, Sonneveld H, van Heerwaarden RJ, Jacobs WC, Wymenga AB. Distal tuberosity osteotomy in open wedge high tibial osteotomy can prevent patella infera: a new technique. Knee, 2004, 11: 457-461.

48. Esenkaya I, Unay K. Proximal medial tibial biplanar retrotubercle open wedge osteotomy in medial knee arthrosis. Knee, 2012, 19: 416-421.

49. Elmalı N, Esenkaya I, Can M, Karakaplan M. Monoplanar versus biplanar medial open-wedge proximal tibial osteotomy for varus gonarthrosis: a comparison of clinical and radiological outcomes. Knee Surg Sports Traumatol Arthrosc, 2013, 21: 2689-2695.

50. Longino PD, Birmingham TB, Schultz WJ, Moyer RF, Giffin JR. Combined tibial tubercle osteotomy with medial opening wedge high tibial osteotomy minimizes changes in patellar height: a prospective cohort study with historical controls. Am J Sports Med, 2013, 41: 2849-2857.

51. Park H, Kim HW, Kam JH, Lee DH. Open Wedge High Tibial Osteotomy with Distal Tubercle Osteotomy Lessens Change in Patellar Position. Biomed Res Int, 2017, 2017: 4636809.

\section{Figures}



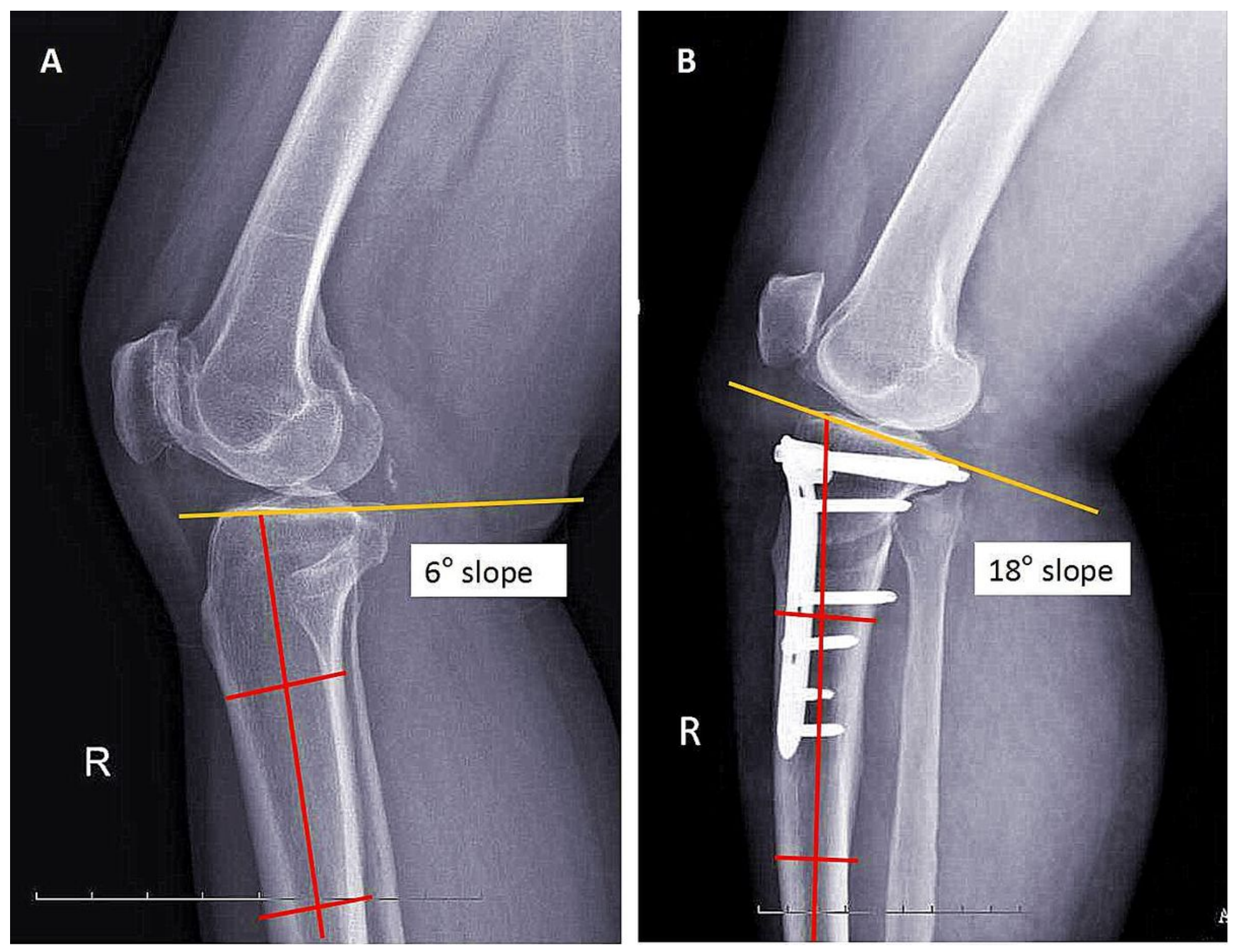

\section{Figure 1}

Lateral radiographs obtained A preoperative and B at 36-month follow-up after OWHTO for medial gonarthrosis of the right knee in a 48-year-old female patient with correction angle of $20^{\circ}$. The PTS is the smaller of the two angles shown and is calculated as $90^{\circ}$ minus the angle created by the proximal tibial anatomic axis and a line tangent to the joint line (medial tibial plateau). 


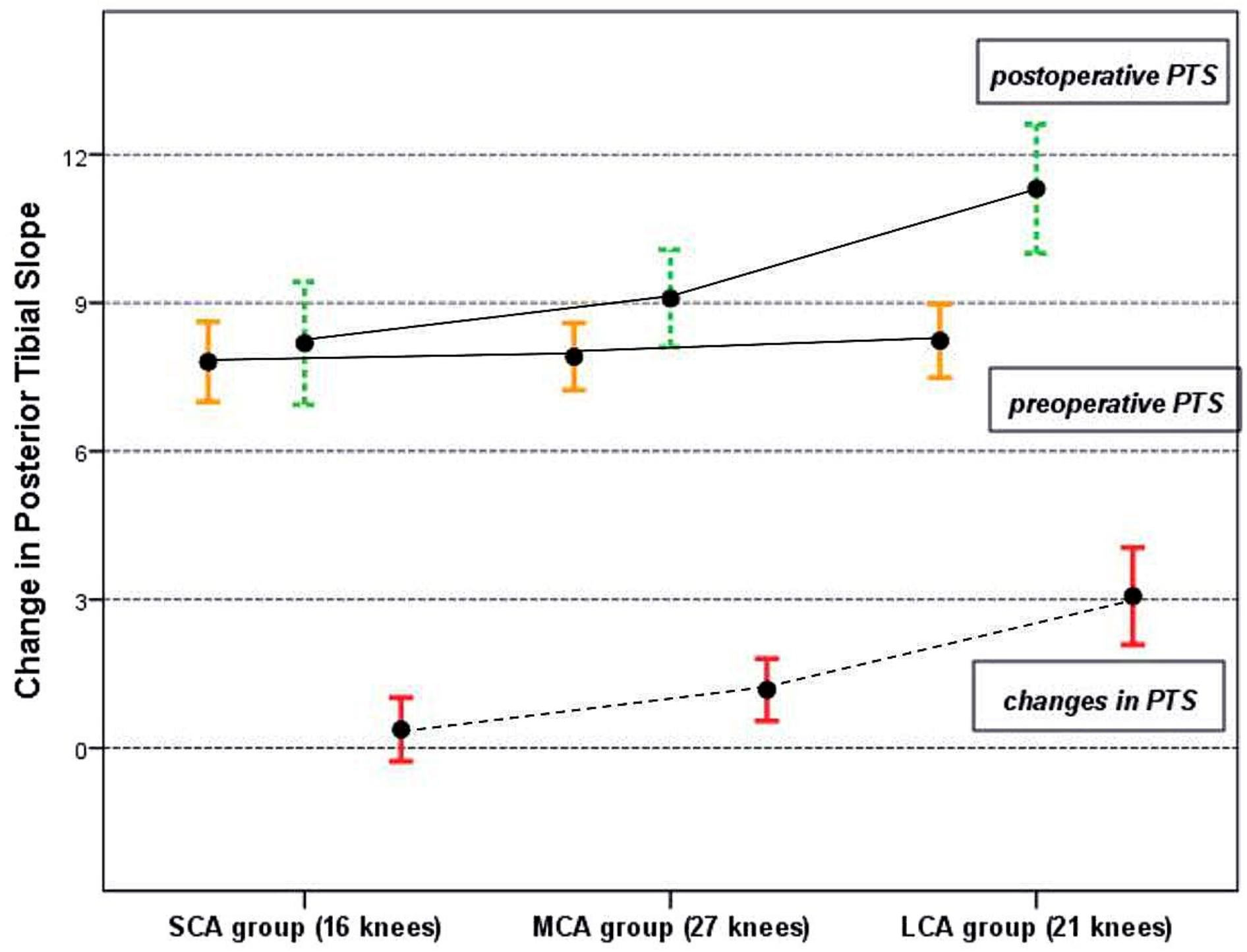

Figure 2

The mean change and $95 \% \mathrm{Cl}$ for the PTS before ans after surgery in each the three groups are shown. 


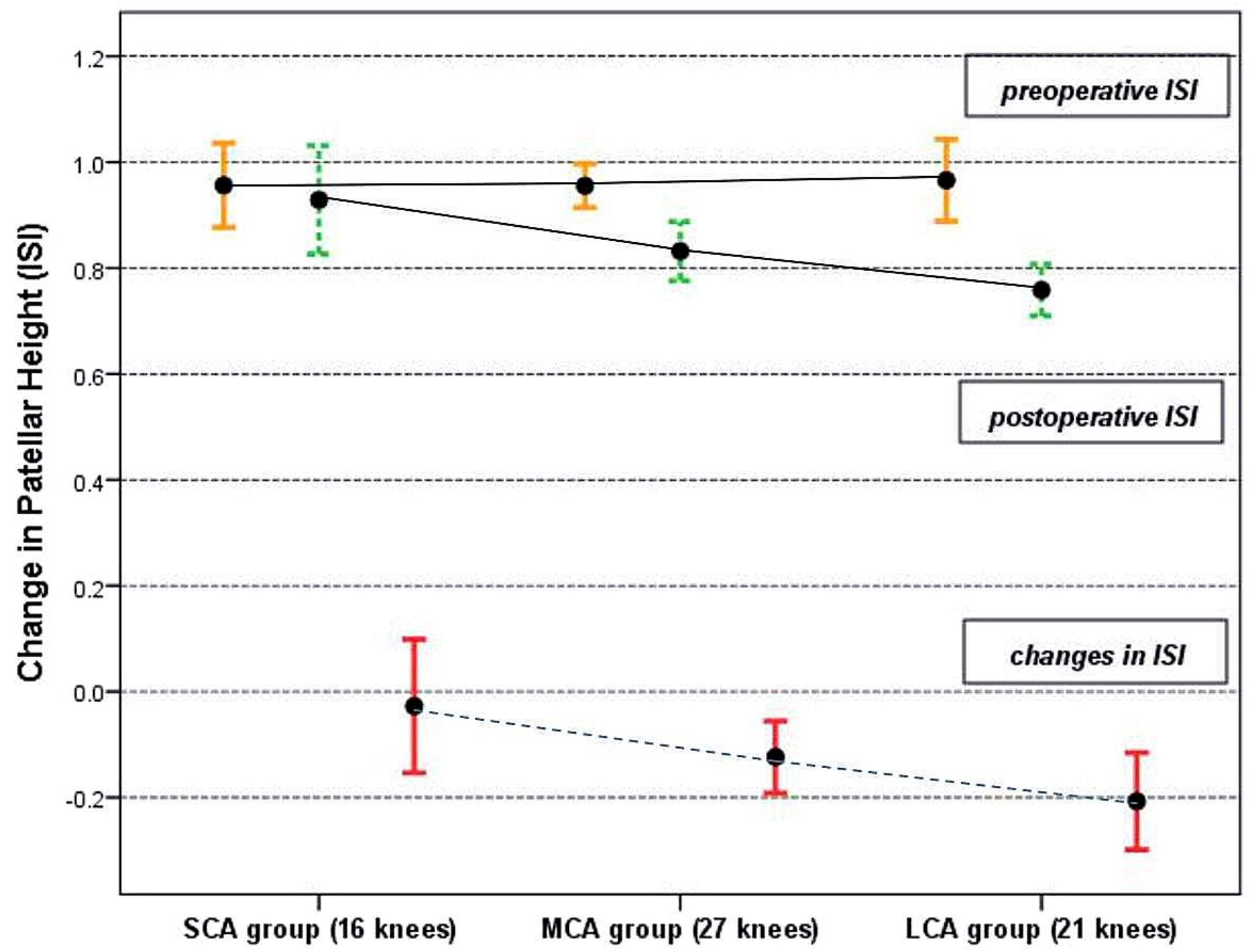

Figure 3

The patellar height of ISI before and after surgery in the three groups with $95 \% \mathrm{Cl}$ are shown. 


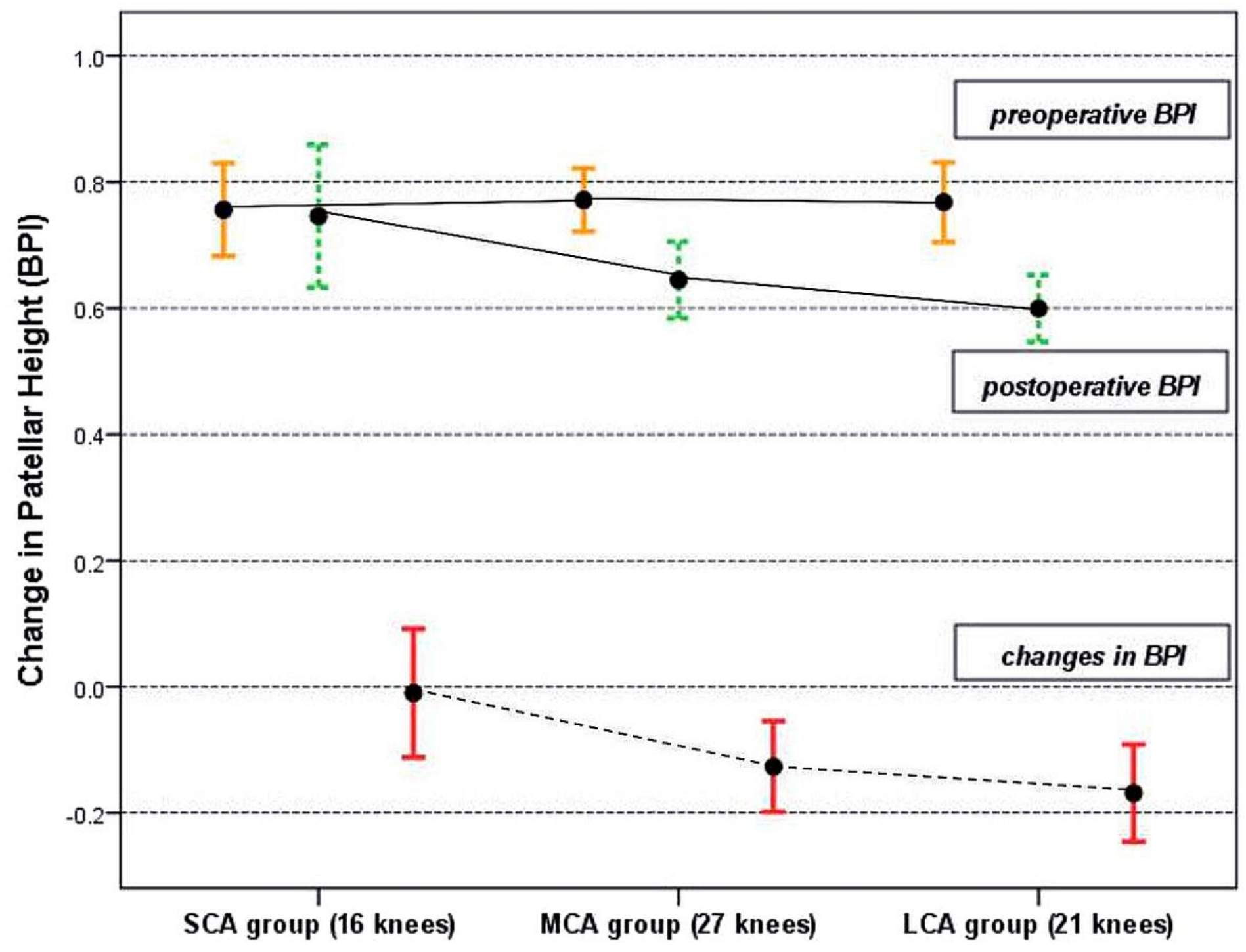

Figure 4

The patellar height of BPI before and after surgery in the three groups with $95 \% \mathrm{Cl}$ are shown. 